\title{
FILOSOFIA DA MENTE, CIÊNCIA COGNITIVA E O PÓS-HUMANO: PARA ONDE VAMOS?, DE MONICA AIUB, MARIA EUNICE QUILICI GONZALEZ E MARIANA CLÁUDIA BROENS (ORGANIZADORAS)
}

\author{
Nivaldo Machado ${ }^{1}$ \\ Elizeu de Oliveira Santos Sobrinho ${ }^{2}$ \\ Universidade Para o Desenvolvimento do Alto Vale do Itajaí (UNIDAVI)
}

\section{AIUB, Monica; GONZALEZ, Maria Eunice Quilici; BROENS, Mariana Cláudia (organizadoras). Filosofia da mente, ciência cognitiva e o pós-humano: para onde vamos? São Paulo: FiloCzar, 2015.}

\section{Estrutura da obra}

A obra é uma homenagem aos sessenta anos de idade do professor João de Fernandes Teixeira, uma das principais referências em Filosofia da Mente e Ciências Cognitivas do país.

Sua estrutura está dividida em três seções. A primeira seção recebe o título "Questões semióticas, epistemológicas e éticas do pós-humano" e conta com os trabalhos de Lúcia Santaella; Ivo Assad Ibri; Mariana Broens, Maria Eunice Quilici Gonzalez, Carmen Beatriz Millidoni e José Arthur Quilici-Gonzalez; Nivaldo Machado; Adriano Neves de Brito; Márcia Avelino; e Monica Aiub.

A segunda seção é intitulada "Reflexões sobre teorias contemporâneas da mente" e recebe os trabalhos de Jonas Gonçalves Coelho; Edna Alves de Souza; Sofia Miguens; Osvaldo Pessoa Jr. e Luma Melo; e Gustavo Leal Toledo.

\footnotetext{
${ }^{1}$ Doutor em Filosofia e Metodologia das Ciências pela Universidade Federal de São Carlos (UFSCAR), São Paulo - Brasil. Coordenador do Grupo de Pesquisa Filosofia da Mente e Ciências Cognitivas. Professor da Universidade Para o Desenvolvimento do Alto Vale do Itajaí, (UNIDAVI) - Brasil. E-mail: nivaldo@unidavi.edu.br

${ }^{2}$ Acadêmico do Curso de Direito da Universidade Para o Desenvolvimento do Alto Vale do Itajaí, (UNIDAVI) - Brasil. Membro do Grupo de Pesquisa Filosofia da Mente e Ciências Cognitivas. E-mail: elizeu.oliveira@unidavi.edu.br
} 
A seção final "Questões atuais de Filosofia da Mente e da Neurociência" recebe os textos de Alfredo Pereira Jr.; Daniel de LucaNoronha; e Gabriel Mogabri.

\section{Questões semióticas, epistemológicas e éticas do pós-humano}

No capítulo que dá início a primeira seção, "O retorno em espiral do pós-humano", a autora Lucia Santaella agrupa as versões interpretativas do pós-humano em quatro grupos, a saber: as versões céticas, apocalípticas, populares e críticas (AIUB; GONZALEZ; BROENS, 2015, p. 21-22) e mostra que Pearson, em sua obra Viroid life (AIUB; GONZALEZ; BROENS, 2015, p. 23), aborda a condição humana por meio de um constante processo de invenção e reinvenção, onde o pensamento deve buscar uma nova conciliação com o aparato tecnológico. Ainda, traz o pensamento de Teixeira de que as linhas divisórias entre o artificial e o humano não resistirão por muito tempo, uma vez que para Teixeira mente e consciência não são privilégios exclusivos dos seres vivos, cabendo assim à feitura de uma nova ciência, sociologia, história e etc., além da novas abordagens sobre questões envolvendo a ética robótica (AIUB; GONZALEZ; BROENS, 2015, p. 25-27).

No segundo capítulo, Ivo Assad Ibri, por meio do texto "Fins vitais e alteridade prática: sobre ciências aplicadas na filosofia de Peirce" (AIUB; GONZALEZ; BROENS, 2015, p. 29) traz, com a filosofia de Peirce como pano de fundo, uma definição de alteridade prática e sua relação com ciências aplicadas, tais como a engenharia, e levanta questionamentos acerca dos compromissos éticos da ciência (AIUB; GONZALEZ; BROENS, 2015, p. 30-34). Consegue aclarar alguns conceitos como ciências aplicadas, tecnologia e técnica (AIUB; GONZALEZ; BROENS, 2015, p. 32-34), para, ao fim, elencar a relação do falibilismo com as ciências aplicadas (AIUB; GONZALEZ; BROENS, 2015, p. 35-37).

O terceiro capítulo "Filosofia da Mente e o pós-humano: para onde vamos com os novos desdobramentos do mecanicismo?", de autoria de Mariana Claudia Broens, Maria Eunice Quilici Gonzalez, Carmem Beatriz Mollidoni e José Arthur Quilici-Gonzalez, tem como principal objetivo investigar possíveis consequências de hipóteses oriundas do paradigma mecanicista sob a ótica da contemporaneidade da mente (AIUB; GONZALEZ; BROENS, 2015, p. 39). Para isso os autores abordam o modelo freudiano de mente que, fundamentado na dinâmica neural, é, em essência, mecânico (AIUB; GONZALEZ; BROENS, 2015, p. 39-40). Indicam como este modelo influenciou e inspirou a Ciência Cognitiva nos vieses da Inteligência Artificial, Conexionismo e da Cognição Situada e Incorporada (AIUB; GONZALEZ; BROENS, 2015, p. 40-43). Com essas abordagens, os autores discutem possíveis implicações éticas de uma

AIUB, Monica; GONZALEZ, Maria Eunice Quilici; BROENS, Mariana Cláudia (organizadoras). Filosofia da mente, ciência cognitiva e o pós-humano: para onde vamos? São Paulo: FiloCzar, 2015. Resenha por Nivaldo Machado e Elizeu de Oliveira Santos Sobrinho 
concepção mecanicista da mente, bem como suas vantagens e desvantagens (AIUB; GONZALEZ; BROENS, 2015, p. 43-45).

Nivaldo Machado é o autor do quarto capítulo. Intitulado "A comunicação no mundo pós-humano: consciência, mentalismo e o artificial" (AIUB; GONZALEZ; BROENS, 2015, p. 49), este capítulo aborda o vocabulário mentalista no mundo pós-humano. $\mathrm{O}$ autor defende a necessidade de um refinamento nas argumentações que buscam delimitar o mundo pós-humano (AIUB; GONZALEZ; BROENS, 2015, p. 50-51). Observa que pelos critérios de economia, adaptação, tempo e explicação, o vocabulário mentalista sustenta sua existência enquanto comunicação entre humanos e suas variabilidades, como robôs, ciborgues e humanoides (AIUB; GONZALEZ; BROENS, 2015, p. 54). Aborda ainda o avanço do mistério da consciência para o problema da consciência, onde conclui que, se aceitos alguns pressupostos, não há problema algum em atribuir consciência a robôs, humanoides e demais seres artificiais (AIUB; GONZALEZ; BROENS, 2015, p. 54-57).

"Responsável, porque determino", de autoria de Adriano Neves de Brito é o quinto capítulo da primeira seção (AIUB; GONZALEZ; BROENS, 2015, p. 59). Logo no início do trabalho o autor deixa claro que seu texto é de cunho naturalista e preza pela reaproximação de diferentes âmbitos do conhecimento como a filosofia, matemática, física, neurociência, psicologia e outros. Sob o enfoque naturalista o autor analisa o problema de responsabilidade e da liberdade na ética, e filia-se a concepção de Crisholm e Strawson sobre liberdade (AIUB; GONZALEZ; BROENS, 2015, p. 5962). Como animais, os seres humanos são suscetíveis às determinações causais que influenciam todos os seres vivos e às determinações que são específicas à espécie humana pelo tipo de comportamento que deve ser realizado em consonância com os reforços oriundos da seleção natural (AIUB; GONZALEZ; BROENS, 2015, p. 62-63). Nesse cenário de determinações, duas são as condições para atribuir responsabilidade a um indíviduo: a) que ele possa agir de outro modo e b) que ele seja suscetível à influência da pressão moral alheia sobre o seu comportamento (AIUB; GONZALEZ; BROENS, 2015, p. 64-65). Tais considerações possuem relevância dentro do sistema jurídico como, por exemplo, no caso de apuração da responsabilidade de menores e de pessoas com danos cerebrais (AIUB; GONZALEZ; BROENS, 2015, p. 65-66).

O penúltimo capítulo da primeira seção é escrito por Márcia Avelino e intitula-se "Dúvidas e dilemas de um ciborgue apaixonado: reflexões éticas sobre os pós-humanos e suas relações" (AIUB; GONZALEZ; BROENS, 2015, p. 69). Utilizando uma afirmação de João de Fernandes Teixeira na obra Inteligência Artificial: uma odisseia da mente a autora aborda as diferentes formas que alguns países a legislação no que se refere aos robôs (AIUB; GONZALEZ; BROENS, 2015, p. 69-70). Em um cenário onde humanos se confundirão com máquinas, criando-se ciborgues, tais

AIUB, Monica; GONZALEZ, Maria Eunice Quilici; BROENS, Mariana Cláudia (organizadoras). Filosofia da mente, ciência cognitiva e o pós-humano: para onde vamos? São Paulo: FiloCzar, 2015. Resenha por Nivaldo Machado e Elizeu de Oliveira Santos Sobrinho 
legislações possuem pertinência uma vez que, em conjunto com a bioética, irão nortear as diversas relações de amizade, afetividade, amor e etc., entre ciborgues de diferentes procedências (AIUB; GONZALEZ; BROENS, 2015, p. 71-75).

Encerrando a primeira seção está o texto de Monica Aiub "Póshumano: um passo no processo evolutivo?" (AIUB; GONZALEZ; BROENS, 2015, p. 79). Utilizando-se dos constructos de João de Fernandes Teixeira, a autora aborda algumas reflexões sobre o pós-humano, em especial, a criação e incorporação de tecnologias no cotidiano humano e como essa relação com a tecnologia acaba por modificar a própria condição humana. Traz a baila questões como: dada essa incorporação de tecnologias, onde irá parar a espécie humana? Como ficarão questões como a discriminação de homens com robôs? (AIUB; GONZALEZ; BROENS, 2015, p. 86-88). Prosseguindo seu trabalho, a autora mostra como os avanços dentro de âmbitos como a Ciência Cognitiva e a Neurociência Cognitiva traçaram uma Filosofia da Neurociência e como essa Filosofia da Neurociência tem na supressão da consciência um importante tópico a ser enfrentado (AIUB; GONZALEZ; BROENS, 2015, p. 80-82).

\section{Reflexões sobre teorias contemporâneas da mente}

A segunda seção é inaugurada por Jonas Gonçalves Coelho com o trabalho "Mente como cérebro e cérebro como mente: a dupla face da relação mente-cérebro" (AIUB; GONZALEZ; BROENS, 2015, p. 93). Neste trabalho o autor traz uma abordagem fisicalista não-reducionista da relação mente-cérebro. Ancorado nos ensinamentos de Searle, Nagel e Chalmers, o autor discute a relação mente-cérebro e o papel da consciência nessa relação, afirmando que há certo exagero no trato com os problemas envolvendo a consciência (AIUB; GONZALEZ; BROENS, 2015, p. 93-95). Apresenta assim uma "abordagem dupla face" da relação mente-cérebro, considerando seriamente os dois lados/direções desta relação mente > cérebro e cérebro > mente (AIUB; GONZALEZ; BROENS, 2015, p. 95). Essa dupla abordagem permite uma solução dentro dos moldes fisicalistas não-reducionistas para o problema da causação mental. Ao invés de perguntar "como a mente, resultante da atividade cerebral, causa um evento físico/cerebral?" deve-se perguntar "como o cérebro, em virtude da mente, age causalmente sobre o físico/cerebral?" (AIUB; GONZALEZ; BROENS, 2015, p. 96-99).

O nono capítulo é de Edna Alves de Souza a apresenta "Uma reflexão sobre o materialismo contemporâneo e a estratégica proposta de um fisicalismo minimalista" (AIUB; GONZALEZ; BROENS, 2015, p. 101). A autora se dedica a uma reflexão acerca das três variedades principais do materialismo (AIUB; GONZALEZ; BROENS, 2015, p. 101-102). Elenca que a primeira variedade, o materialismo eliminativo ou monismo

AIUB, Monica; GONZALEZ, Maria Eunice Quilici; BROENS, Mariana Cláudia (organizadoras). Filosofia da mente, ciência cognitiva e o pós-humano: para onde vamos? São Paulo: FiloCzar, 2015. Resenha por Nivaldo Machado e Elizeu de Oliveira Santos Sobrinho 
materialista, é a tese de que o corpo e o que se entende por mente são um único elemento cuja natureza é material, assim, não existiria qualquer outra coisa além de sistemas físicos (AIUB; GONZALEZ; BROENS, 2015, p. 102-103). O materialismo redutivo (teoria da identidade mente-cérebro) é a doutrina que defende que tudo o que existe poderia ser reduzido à matéria, de modo que uma teoria da identidade pode ser caracterizada por identificar estado mental a estado cerebral (AIUB; GONZALEZ; BROENS, 2015, p. 103). Por sua vez, o materialismo emergente (dualismo de propriedade) defende a tese de que a única substância existente é a material, mas essa substância possui propriedades físicas e outras não-físicas, estas substâncias não-físicas não podem ser explicadas em termos físicos, mesmo tendo emergido de uma substância física (AIUB; GONZALEZ; BROENS, 2015, p. 104) Por último, mostra o fisicalismo minimalista proposto por Teixeira, que tem como um dos principais objetivos eliminar a ideia de que tudo deva ser material ou imaterial uma vez que, segundo o fisicalismo minimalista, a mente é distinta da matéria, todavia, não deixa de ser um fenômeno natural (AIUB; GONZALEZ; BROENS, 2015, p. 104-107).

O décimo capítulo recebe o título "Encontros comigo mesmo: reflexões sobre subjetividade, consciência e linguagem em torno de Donald Davidson e Shaun Gallagher" e é elaborado por Sofia Miguens (AIUB; GONZALEZ; BROENS, 2015, p. 109). A autora mostra de diferentes perspectivas o lugar, papel e importância da linguagem e da consciência dentro do estudo da subjetividade (AIUB; GONZALEZ; BROENS, 2015, p. 109-110). Para isso utiliza os trabalhos de Donald Davidson e Shaun Gallagher. Davidson apresenta uma proposta envolta em um projeto de uma teoria geral das relações pensamento-linguagem-mundo, que traz a subjetividade como autoridade de primeira pessoa (AIUB; GONZALEZ; BROENS, 2015, p. 111-113). Gallagher analisa o fenômeno da imunidade (ou não imunidade) aos erros de identificação (IEM) e defende que a perspectiva de primeira pessoa é um ponto de origem, um esquema corporal não relativo, integrado com o quadro espacial egocêntrico de referência (AIUB; GONZALEZ; BROENS, 2015, p. 114-117).

"O dualismo interacionista não precisa violar leis de conservação da física" é o décimo primeiro capítulo (AIUB; GONZALEZ; BROENS, 2015, p. 119). Osvaldo Pessoa Jr. e Luma Melo mostram que a concepção cartesiana de que a mente e o corpo são substâncias distintas e independentes que interagem por meio da glândula pineal ou "glândula interacionista", não implica, necessariamente, na assertiva de que as teorias dualistas (incluído o dualismo cartesiano) violam o princípio de conservação de energia (AIUB; GONZALEZ; BROENS, 2015, p. 119-121). Para isso eles utilizam como modelo da glândula interacionista (ou glândula pineal, como propunha Descartes) o "aniquilamento de duas partículas e a subsequente geração de um par de raios gama" (AIUB; GONZALEZ; BROENS, 2015, p. 119).

AIUB, Monica; GONZALEZ, Maria Eunice Quilici; BROENS, Mariana Cláudia (organizadoras). Filosofia da mente, ciência cognitiva e o pós-humano: para onde vamos? São Paulo: FiloCzar, 2015. Resenha por Nivaldo Machado e Elizeu de Oliveira Santos Sobrinho 
O capítulo que encerra a segunda seção é de Gustavo Leal Toledo e intitula-se "O paradoxo de Chalmers" (AIUB; GONZALEZ; BROENS, 2015, p. 123). O autor expõe de maneira breve o argumento dos zumbis proposto por David Chalmers e traz as críticas sofridas por este argumento no que se refere ao "problema das outras mentes" e o "problema da minha mente" (AIUB; GONZALEZ; BROENS, 2015, p. 123-125). Para responder as críticas, Chalmers utiliza o chamado paradoxo do julgamento fenomenal e defende que a consciência seria uma espécie de prova de si mesma, onde não é possível dizer se ele é consciente ou não, mas é possível dizer que $\mathrm{eu}$ sou consciente (AIUB; GONZALEZ; BROENS, 2015, p. 124-125). A partir daí o autor crítica os constructos de Chalmers de que a consciência é um explanandum inegável (AIUB; GONZALEZ; BROENS, 2015, p. 125). Argumenta que para Chalmers levar a consciência a sério é reconhecer o paradoxo e aprender e conviver com ele, uma vez que a consciência é prova de si mesma (AIUB; GONZALEZ; BROENS, 2015, p. 126-129). Conclui que, apesar de ser idealizado a favor do dualismo, o argumento dos zumbis acaba voltando-se contra si mesmo quando possibilita o surgimento da dúvida em relação aos qualia (AIUB; GONZALEZ; BROENS, 2015, p. 130-131).

\section{Questões atuais de Filosofia da mente e Neurociência}

A terceira seção tem início com Alfredo Pereira Júnior com o capítulo "Breve revisão das hipóteses sobre o código neuronal" (AIUB; GONZALEZ; BROENS, 2015, p. 135). De início o autor elenca os "principais temas epistemológicos que resultam da discussão de um código neuronal e da natureza do processamento da informação no cérebro." (AIUB; GONZALEZ; BROENS, 2015, p. 135). Um dos pontos principais no estudo do código neuronal é compreender como os neurônios centrais trabalham (AIUB; GONZALEZ; BROENS, 2015, p. 137). Compreendido seu funcionamento, seria possível estreitar a relação entre redes neuronais artificiais e estruturas neuronais biológicas (Neurociência Computacional) (AIUB; GONZALEZ; BROENS, 2015, p. 137-139). Ainda referindo-se aos códigos neuronais, o autor cita duas propostas clássicas, a ideia de um código de população (Adrian) e a ideia de que a codificação seria feita através de neurônios individualmente especializados (Barlow) (AIUB; GONZALEZ; BROENS, 2015, p. 139-140). Por fim, apresenta a proposta de um código temporal em contraste com a proposta de codificação espacial/populacional (AIUB; GONZALEZ; BROENS, 2015, p. 141-143).

$\mathrm{O}$ décimo quinto capítulo "Reconhecer crenças falsas sem metarrepresentações" é de autoria de Daniel De Luca-Noronha (AIUB; GONZALEZ; BROENS, 2015, p. 145). De início o autor mostra que o reconhecimento de crenças falsas é importante para o desenvolvimento social dos seres humanos (AIUB; GONZALEZ; BROENS, 2015, p. 145).

AIUB, Monica; GONZALEZ, Maria Eunice Quilici; BROENS, Mariana Cláudia (organizadoras). Filosofia da mente, ciência cognitiva e o pós-humano: para onde vamos? São Paulo: FiloCzar, 2015. Resenha por Nivaldo Machado e Elizeu de Oliveira Santos Sobrinho 
Mostra o contraste dos experimentos conduzidos por Perner e Wimmer com os experimentos das psicólogas Baillargeon e Onishi e como estes experimentos fazem parecer que o reconhecimento de crenças falsas depende da capacidade de confrontar perspectivas opostos sobre o mundo, o que requereria certo nível de "flexibilidade cognitiva", encontrado na posse explícita de metarrepresentações (AIUB; GONZALEZ; BROENS, 2015, p. 146-148). Essa "flexibilidade cognitiva" é investigada para saber se existiria de forma independente de metarrepresentações (AIUB; GONZALEZ; BROENS, 2015, p. 148-152).

"Algumas coisas que acho que sei sobre o pensamento do Professor João de Fernandes Teixeira", de Gabriel Mogabri, é o capítulo que encerra a seção e a obra (AIUB; GONZALEZ; BROENS, 2015, p. 155). Sob a ótica do autor, o pensamento do Professor João de Fernandes Teixeira é exposto. De início é elencado o posicionamento materialista não-redutivista do Professor João, assim como seu ceticismo no que se refere ao uso de mecanismos quantitativos empíricos para o estabelecimento de conteúdo mentais e a preferência pelo viés da simulação computacional, inteligência artificial e etc. (AIUB; GONZALEZ; BROENS, 2015, p. 155-156). Ressalta a interessante discussão que teve com o homenageado no XIV Encontro Nacional da ANPOF, em 2010 (AIUB; GONZALEZ; BROENS, 2015, p. 156-159). Por fim, em tom de agradecimento, ressalta o "caráter extremamente democrático, republicano e aberto da postura de debate" do Professor João de Fernandes Teixeira (AIUB; GONZALEZ; BROENS, 2015, p. 160).

\section{Contribuição dos autores:}

Os autores Nivaldo Machado e Elizeu de Oliveira Santos Sobrinho participaram da leitura, revisão e redação do artigo. Ambos aprovaram a versão final do texto.

AIUB, Monica; GONZALEZ, Maria Eunice Quilici; BROENS, Mariana Cláudia (organizadoras). Filosofia da mente, ciência cognitiva e o pós-humano: para onde vamos? São Paulo: FiloCzar, 2015. Resenha por Nivaldo Machado e Elizeu de Oliveira Santos Sobrinho 